Proceedings of the XXIII Conference on Applied Crystallography, Krynica Zdrój, Poland, September 20-24, 2015

\title{
Calculations of RHEED and RHEPD Rocking Curves for Growing Surfaces of Germanium
}

\begin{abstract}
Z. MiturA*
AGH University of Science and Technology, Faculty of Metals Engineering and Industrial Computer Sci., al. A. Mickiewicza 30, 30-059 Kraków, Poland

Reflection high energy electron diffraction is a popular technique to characterize arrangements of atoms near a surface. However, Japanese researchers recently demonstrated experiments in the same geometry, however, conducted using positrons. In this context, detailed comparisons of basic results expected for diffractions of electrons and positrons seem to be interesting. Subsequently, in the current work the growth of single atomic layers of $\mathrm{Ge}$ on the $\mathrm{Ge}(001)$ substrate is assumed and intensities of reflected beams for electrons and positrons are computed by using dynamical diffraction theory for the case of the off-symmetry azimuth. Shapes of respective theoretical rocking curves are analyzed and then the features of intensity oscillations expected during the regular, continuous deposition of the material are discussed.
\end{abstract}

DOI: 10.12693/APhysPolA.130.1134

PACS/topics: 61.05.J-, 68.35.B-, 81.05.Cy

\section{Introduction}

Information on the arrangement of atoms near the surface is very important for scientists dealing with the preparation of nanostructures on solid-state substrates under ultra-high vacuum conditions. Reflection high energy electron diffraction (RHEED) can be applied to obtain information of this type [1]. In respective experiments, electrons strongly interact with atoms at the surface and effectively penetrate only the region with the thickness of about 10-30 $\mathrm{nm}$ along the axis perpendicular to the surface. For diffraction of electrons, advanced theories should be used to interpret experimental results quantitatively. In fact, the research work should be carried out to develop precise theoretical approaches for analyses of data for imperfect surfaces, see for example [2]. Nevertheless, it can be said in some simplification that RHEED is a relatively mature technique.

However, in the last years it has been demonstrated in a series of publications that positrons can be also used to obtain information on the arrangements of atoms at the surface. Herein we recall only basic facts on reflection high energy positron diffraction (RHEPD), for a more detailed account see the review paper of Fukaya et al. [3]. Namely, the first experiments were conducted in the late 1990s [4]. Earlier, Ichimiya published the theoretical paper in which possible advantages of this technique were described [5]. Actually, the basic obstacle in conducting RHEPD experiments is the requirement to form a coherent incident beam. Satisfying this condition for positrons is much more difficult than for electrons. Thus, until now the experimental work for RHEPD was conducted only in Japan. In the first experiments, a ${ }^{22} \mathrm{Na}$ source allowed

*e-mail: mitura@metal.agh.edu.pl researchers to obtain a small number of positrons [4]. However, a new experimental setup has been recently developed where the incident positrons are formed with the help of a special line accelerator [6], which extends possibilities for conducting interesting experiments. It is important to mention here that in some situations the surface sensitivity for RHEPD is expected to be substantially greater than that for RHEED. This is a consequence of the effect of the total reflection in the case of RHEPD [5, 6]. For reflected electrons, this effect does not occur. It is also worth to add that for X-rays, the situation is generally similar to that of positrons, however, the effect of total reflection for X-rays occurs only in a very narrow angular range.

\section{Calculations}

For undertaking described research work it was of the particular importance that RHEED and RHEPD computations can be executed using the same numerical code developed within dynamical diffraction theory using twodimensional Bloch wave approach [5]. Moreover, to run the calculations for positrons, one can use the same computer program as for electrons when changing the sign of the real part of the scattering potential to account the change of the particle charge [7]. It seems interesting to compare theoretical results for RHEED and RHEPD for assumed continuous deposition of material on a substrate. This is because RHEED is quite often used to monitor changes at the surface during such depositions. Concerning RHEPD, its usage in the experimental work of this type has not been reported so far, however, one may expect that this happens in the near future.

We assumed a perfect layer by layer growth of $\mathrm{Ge}(001)$ layers on a $\mathrm{Ge}(001)$ substrate. It means that during the growth only one incomplete atomic layer exists at the surface. Intensities of reflected electrons and positrons were calculated as described in [7]. Namely, for full layers, the scattering potential was averaged in the $z$-planes 
(i.e. we carried out "one beam case" calculations [1]). Additionally, it was assumed that the scattering potential of an incomplete, atomic layer is proportional to its coverage $\theta$ and the potential of the fully completed layer (for a discussion of this assumption see for example [8]). The energy of electrons and positrons was taken to be $15 \mathrm{keV}$.

\section{Results}

Figure 1 shows rocking curves, i.e. plots of intensities of the specularly reflected beam for different glancing angles of the incident beam. If we take a look at Fig. 1, we can recognize that in general, shapes of the rocking curves for RHEED and RHEPD are similar. However, due to the effect of refraction, in both cases positions of the Bragg peaks are modified. For RHEED, the peaks are moved towards lower angles. For RHEPD, the peaks are shifted into the opposite direction, i.e. towards higher angles. However, for RHEPD additionally there occurs an effect of the total reflection for angles smaller than the critical angle $\theta_{c}$. We can find the value of $\theta_{c}$ if we follow the prescription given by Ichimiya [5]. Namely, the condition of the total reflection for the case of RHEPD can be written as follows: $\sin ^{2} \theta_{c}=v_{a v} / K^{2}$, where $\theta_{c}$ is the critical angle, $K$ is magnitude of the wave vector and $v_{a v}$ is the volume average potential inside the crystal bulk [7]. For the assumed energy of $15 \mathrm{keV}$, the value of $K^{2}$ is equal to $3994.3 \AA^{-2}$. Further, for the crystal of

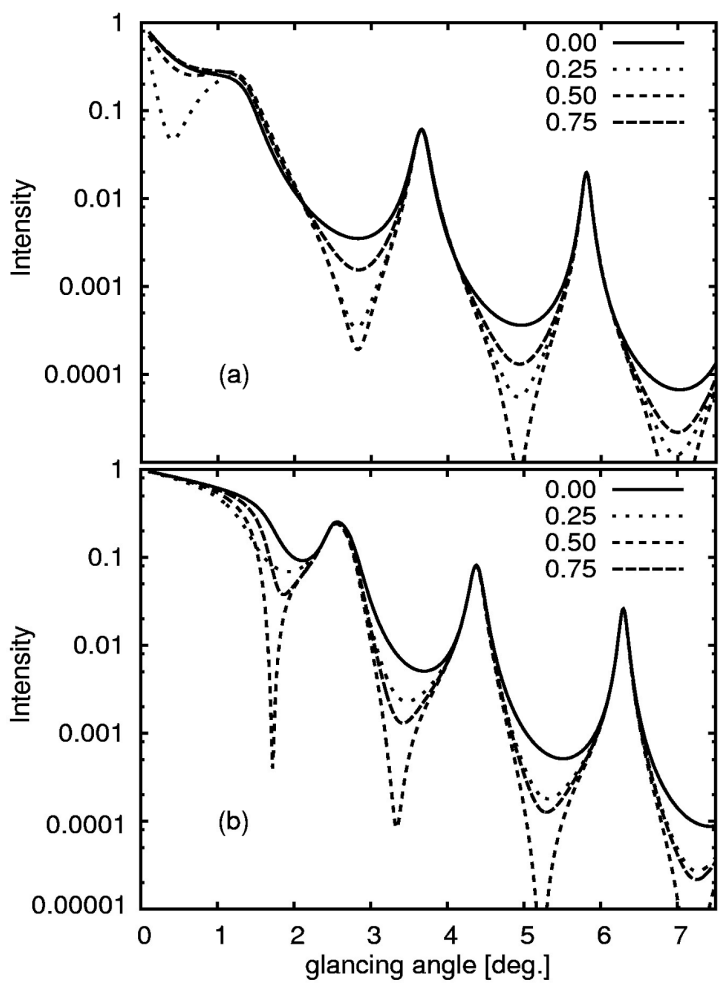

Fig. 1. A series of RHEED and RHEPD rocking curves for the surface of Ge (001) for different coverages $\theta$ of the growing layer (values of $\theta$ are given in the figure). Curves for RHEED and RHEPD are shown respectively in parts (a) and (b).

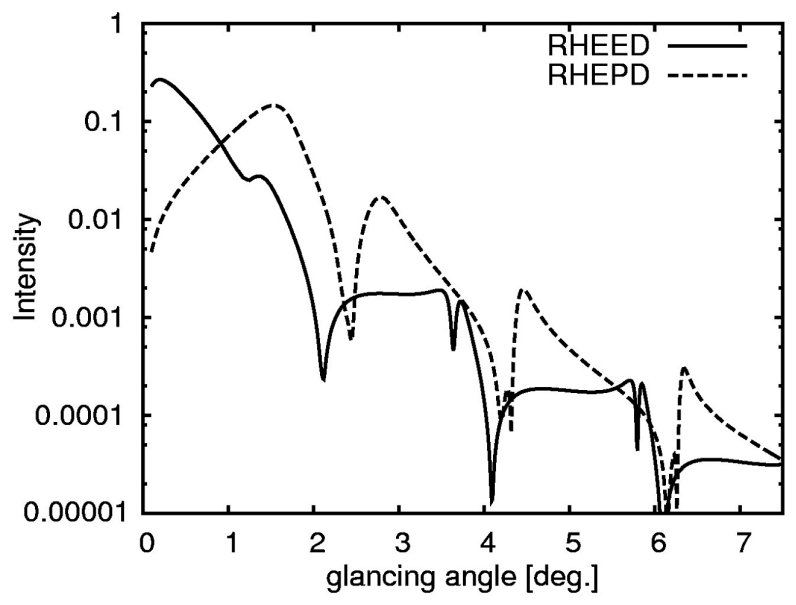

Fig. 2. Amplitudes of changes in the RHEED and RHEPD intensity during the deposition of a new layer of the material.

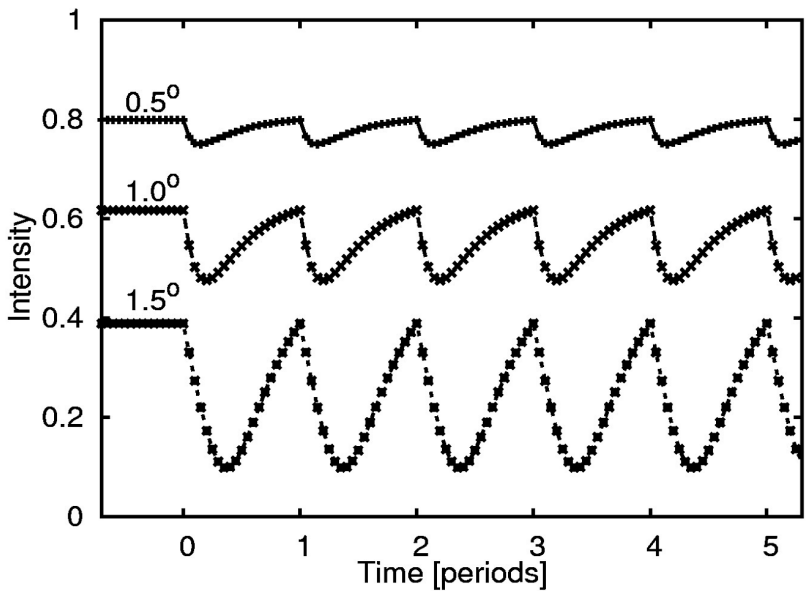

Fig. 3. Runs of RHEPD oscillations determined computationally for different glancing angles $\theta$ below the critical angle $\theta_{c}$ (values of $\theta$ are given in the Figure).

Ge(001) we apply: $v_{a v}=3.5964 \AA^{-2}$. Therefore $\theta_{c}$ is equal to $1.72^{\circ}$.

It is widely known that RHEED can be used to observe changes in the intensity during the deposition of material at the substrate. If the growth is very regular then the oscillating changes occur with the period corresponding to the deposition of a single atomic layer [1]. RHEED oscillations were first observed in early 1980s. So far, RHEPD observations accompanying the growth of thin films were not reported. However, in any case, it is interesting to compare theoretically expected amplitudes of RHEED and RHEPD oscillations during the deposition of Ge (see Fig. 2). One can observe that, for RHEED, oscillations with the largest amplitudes are expected for very small glancing angles. However, for RHEPD, the largest amplitudes of intensity changes are for the angles close to the critical angle $\theta_{c}$. 
In general, both for RHEED and RHEPD, the times of the appearance of minima and maxima in oscillations runs depend on a selected value of the glancing angle of the primary beam. However, it is interesting to take a closer look for RHEPD at the intensity oscillations which one should expect for angles lower than the critical angle $\theta_{c}$ (see Fig. 3). One can observe that the minima may occur for different coverages of the growing layer, but the maxima of the intensity always occur when the growing layer is completed. It seems that this observation may be helpful in the future for researchers who prepare thin films. This is because the precise determination of the time when the layer is completed is very important for technology of thin film growth [9].

\section{Acknowledgments}

This research was supported by AGH Project No. 11.11.110.291. The author is very grateful to Professor Ayahiko Ichimiya for his providing information on RHEPD experiments.

\section{References}

[1] A. Ichimiya, P.I. Cohen, Reflection High Energy Electron Diffraction, Cambridge University Press, Cambridge 2004.

[2] J. Huang, C.Y. Cai, C.L. Lv, G.W. Zhou, Y.G. Wang, Philos. Mag. 95, 4095 (2015).

[3] Y. Fukaya, M. Maekawa, I. Mochizuki, K. Wada, T. Hyodo, A. Kawasuso, J. Phys. Conf. Ser. 443, 012068 (2013).

[4] A. Kawasuso, S. Okada, Phys. Rev. Lett. 81, 2695 (1998).

[5] A. Ichimiya, Solid State Phenom. 28-29, 143 (1992).

[6] Y. Fukaya, M. Maekawa, A. Kawasuso, I. Mochizuki, K. Wada, T. Shidara, A. Ichimiya, T. Hyodo, Appl. Phys. Expr. 7, 056601 (2014).

[7] Z. Mitura, Acta Crystallogr. A 71, 513 (2015).

[8] Z. Mitura, S.L. Dudarev, M.J. Whelan, J. Cryst. Growth 198-199, 905 (1999).

[9] M.C. Sullivan, M.J. Ward, A. Gutiérrez-Llorente, E.R. Adler, H. Joress, A. Woll, J.D. Brock, Appl. Phys. Lett. 106, 031604 (2015). 